\title{
A New Fast Nonsingular Terminal Sliding Mode Control for a Class of Second-Order Uncertain Systems
}

\author{
Linjie Xin, Qinglin Wang, and Yuan Li \\ School of Automation, Beijing Institute of Technology, Zhongguancun Street, Haidian District, Beijing, China \\ Correspondence should be addressed to Yuan Li; liyuan@bit.edu.cn
}

Received 12 July 2016; Revised 30 October 2016; Accepted 15 November 2016

Academic Editor: Asier Ibeas

Copyright (C) 2016 Linjie Xin et al. This is an open access article distributed under the Creative Commons Attribution License, which permits unrestricted use, distribution, and reproduction in any medium, provided the original work is properly cited.

\begin{abstract}
This paper considers the robust and adaptive nonsingular terminal sliding mode (NTSM) control for a class of second-order uncertain systems. First, a new fast NTSM was proposed which had global fast convergence rate in the sliding phase. Then, a new form of robust NTSM controller was designed to handle a wider class of second-order uncertain systems. Moreover, an exponential-decline switching gain was introduced for chattering suppression. After that, a double sliding surfaces control scheme was constructed to combine the NTSM control with the adaptive technique. The benefit is that a strict demonstration can be given for the stagnation problem in the stability analysis of NTSM. Finally, a case study for tracking control of a variable-length pendulum was performed to verify the proposed controllers.
\end{abstract}

\section{Introduction}

It is well known that the sliding mode control (SMC) has invariant property to the matched uncertainties. It has become a popular method to control nonlinear uncertain systems $[1,2]$. In the earlier studies of SMC, the research was focused on the linear sliding mode (LSM). In [3, 4], the concept of terminal attractor was proposed and used to design the sliding mode which was known as the terminal sliding mode (TSM). The TSM had advantage of finite time convergence, and it has been widely used in many applications, such as robots, spacecraft, and DC-DC buck converters [5-7].

Compared to the LSM, the TSM has lower convergence speed when the states are far from the origin. Considering this problem, Yu et al. designed a fast terminal sliding mode (FTSM) which had global fast convergence in the sliding phase [8]. The FTSM technique has been developed for controlling nonlinear second-order systems with uncertain terms in [9-11]. Moreover, the concept of terminal attractor was also applied to the reaching law by Yu and Man [12]. As the negative fractional power exists in the TSM control signals, it may result in singularity of the control input. This is hard to accept in real implementations. So, the control input was switched to a general sliding mode control in order to avoid the singularity when the states converged into a small vicinity of origin in [13]. However, this switching method is a suboptimal solution which lost the advantage of TSM. In $[14,15]$, a new form of TSM known as nonsingular TSM (NTSM) was proposed and applied to robotic manipulators and piezoelectric actuators. It also has a lower convergence speed when the states are far from the origin. Although the NTSM control has solved the singularity problem, it needs an extra demonstration to show that the motion of sliding surface will not stagnate at some nonzero points in the reaching phase. According to the existing literatures, most of the TSM control methods were designed for the second-order systems. In [16-18], the recursive TSM control was developed for higher order nonlinear systems.

For the robust TSM controllers, the boundary information of system uncertainties is usually required to be known in advance. However, it is difficult to obtain in many practical implementation processes. Therefore, the adaptive technique was employed to cooperate with the TSM and FTSM control in [19-21]. Moreover, the adaptive NTSM control method was developed for uncertain systems with input nonlinearity in $[22,23]$. Note that a strict proof for the stagnation problem of NTSM can be given in the robust NTSM control, for example, 
$[14,15]$. However, it was difficult to obtain for the case of adaptive NTSM control in [22, 23].

Previous studies have developed the TSM control and thus have great significance. On the other hand, there are still some points valuable for further research. Most of the NTSM controllers were designed for a class of uncertain systems in which the coefficient of control input was a known function (or with an uncertain item). In the case that this coefficient is totally unknown except its boundary, few NTSM controllers were reported. So, the purpose of this paper is to develop the NTSM control for the latter case.

The main contribution of this paper can be summarized as follows.

(1) A fast NTSM (FNTSM) is designed to combine the advantages of LSM and NTSM together. Thus, the convergence rate of the NTSM is enhanced when the initial states are far away from the origin.

(2) A new robust NTSM controller is proposed for the case that the coefficient of control input is unknown. It is also applicable to the case that the coefficient is known. Moreover, an exponential-decline switching gain is designed to attenuate the chattering phenomenon.

(3) An adaptive NTSM control scheme with double sliding surfaces is proposed to give a strict proof for the stagnation problem of NTSM. The system uncertainty is firstly compensated on an integral sliding surface; after that the system trajectory is forced to the designed fast NTSM.

\section{Robust NTSM Controller Design}

Consider a second-order nonlinear system as

$$
\begin{aligned}
& \dot{x}_{1}=x_{2}, \\
& \dot{x}_{2}=\varphi(x)+\gamma(x) u,
\end{aligned}
$$

where $\varphi(x)$ and $\gamma(x)$ denote bounded uncertain functions, $\gamma(x) \neq 0$.

Assumption 1. The uncertain functions $\varphi(x)$ and $\gamma(x)$ satisfy

$$
\begin{aligned}
|\varphi(x)| & \leq \bar{\varphi}=\varphi_{0}+\varphi_{1}\left|x_{1}\right|+\varphi_{2}\left|x_{2}\right|, \\
0 & <K_{m} \leq \gamma(x) \leq K_{M},
\end{aligned}
$$

where $\varphi_{0}, \varphi_{1}, \varphi_{2}, K_{m}$, and $K_{M}$ are positive constants. $K_{m}$ and $K_{M}$ are assumed to be known. Robust and adaptive controllers are designed for the two cases that $\bar{\varphi}$ is known or not, respectively. In the rest of the paper, $\varphi(x)$ and $\gamma(x)$ are simply denoted as $\varphi$ and $\gamma$.

2.1. FNTSM Design. In the study of [14], a kind of NTSM was designed as

$$
\begin{aligned}
& s=x_{1}+\beta\left|x_{2}\right|^{\chi} \operatorname{sign}\left(x_{2}\right), \\
& \qquad x_{2}=\dot{x}_{1}, 0<\beta, 1<\chi<2 .
\end{aligned}
$$

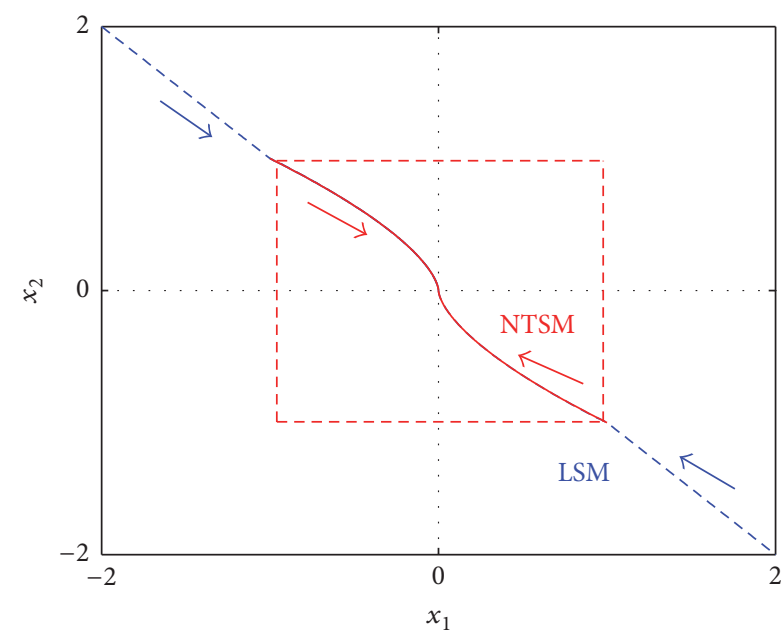

FIGURE 1: The proposed sliding surface on the phase plane.

It is equivalent to the following form which can be converted to a conventional TSM:

$$
s= \begin{cases}x_{1}+\beta x_{2} \chi & x_{2}>0 \\ x_{1} & x_{2}=0 \\ x_{1}-\beta\left(-x_{2}\right)^{\chi} & x_{2}<0 .\end{cases}
$$

As illustrated in [8], the TSM has slower convergence rate than the LSM when the initial states are far away from the origin. It can be explained by its eigenvalue. For TSM $\dot{x}_{1}+$ $\beta^{-1 / \chi} x_{1}^{1 / \chi}=0$, it is easy to obtain the eigenvalue as

$$
\frac{\partial \dot{x}_{1}}{\partial x_{1}}=-\beta^{-1 / \chi} \frac{1}{\chi} x_{1}^{1 / \chi-1} .
$$

As $1 / \chi-1<0$, the eigenvalue tends to be negative infinity at the origin. This fact implies that the convergence rate is infinitely large. This is the major merit of TSM. However, when the initial state is far away from the origin, the convergence rate is smaller than the case $\chi=1$, that is, a LSM.

In order to enhance the convergence rate of NTSM (3), a new FNTSM is designed as

$$
\begin{aligned}
s= & x_{1}+\beta\left|x_{2}\right|^{q} \operatorname{sign}\left(x_{2}\right), \\
& q=\delta+(1-\delta) \operatorname{sign}\left(\left|x_{1}\right|-\varepsilon\right), \quad 1 \leq \varepsilon, 1<\delta<1.5 .
\end{aligned}
$$

The FNTSM (6) is a switching sliding surface which transfers the system dynamic from a LSM $(q=1)$ to a NTSM $(1<q<2$ ) (Figure 1; e.g., $\varepsilon=1$ ). Thus, it has global fast convergence rate and reserves the advantage of NTSM.

Here, a simple example is given to compare the NTSM (3) and the FNTSM (6),

$$
\begin{aligned}
& \dot{x}_{1}=x_{2}, \\
& \dot{x}_{2}=u .
\end{aligned}
$$




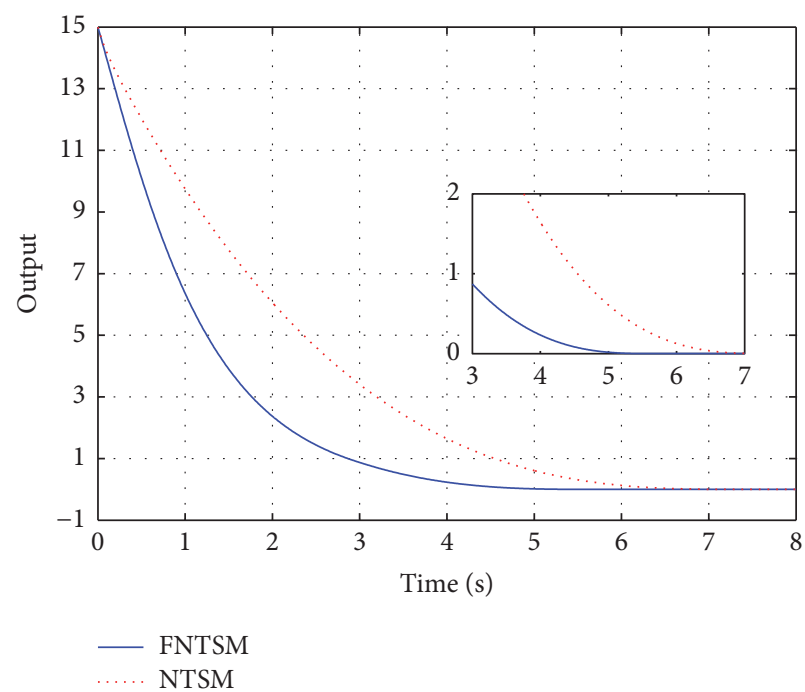

(a) System response

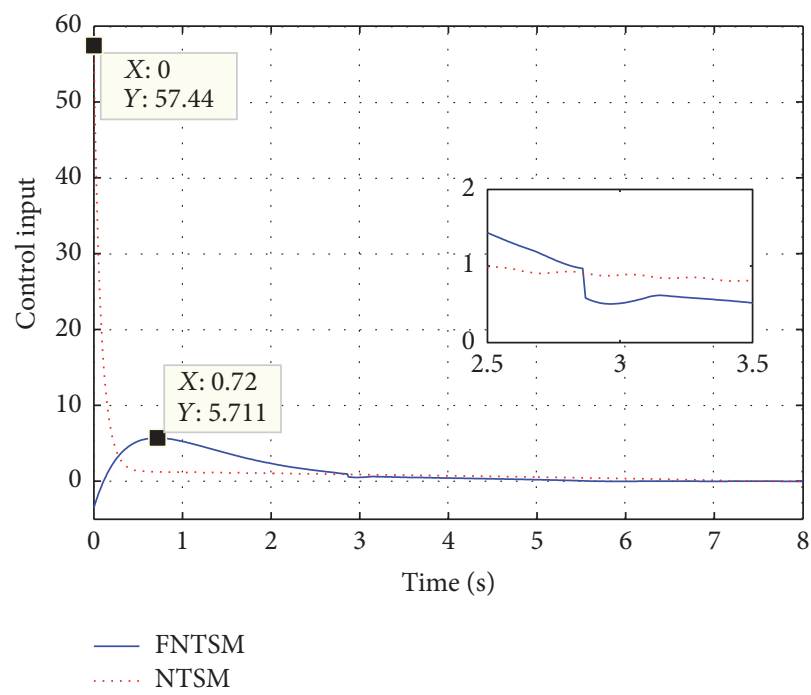

(b) Control input

FIGURE 2: Comparison between the proposed FNTSM and the NTSM.

Considering system (7), the control input was taken as the NTSM controller form of [14]

$$
u=-\frac{1}{q}\left|x_{2}\right|^{2-q} \operatorname{sign}\left(x_{2}\right)-2 s-|s|^{7 / 9} \operatorname{sign}(s) .
$$

With the same reaching law, the parameters for the sliding surfaces were chosen as

$$
\begin{aligned}
& \text { NTSM: } q=q_{N}=\frac{11}{7}, \\
& \text { FNTSM: } q=q_{F}=\frac{9}{7}+\left(1-\frac{9}{7}\right) \operatorname{sign}\left(\left|x_{1}\right|-1\right) .
\end{aligned}
$$

Note that the FNTSM became equivalent to the NTSM within the region $\left|x_{1}\right| \leq 1$ because of $q_{F}=11 / 7$ inside this region. As shown in Figure 2, the convergence rate of the proposed FNTSM controller was faster than the NTSM controller's, while the control input of FNTSM controller was much smaller. In particular, for the FNTSM (6), the switching of sliding surface is not continuous. When the state $x_{1}$ converged into the prescribed region, the sliding surface and control signal had slight discontinuous changes. As a result, the control signal switched to a NTSM control from a LSM control. As shown in Figure 2(b), there was a small saltation of the control signal (from 0.967 to 0.582 ) at $t=$ $2.86 \mathrm{~s}$ because of the switching of sliding surface.

2.2. Controller Design. Most of the NTSM controllers were designed for system (1) with a known $\gamma$ (or with an uncertain item). However, these controllers are not theoretically applicable to totally unknown $\gamma$. In this subsection, a new form of NTSM controller is designed for system (1) in the case that $\bar{\varphi}$ is known. A proof is given to show that the stagnation problem of sliding surface will not occur. Moreover, the new NTSM controller is also applicable to the case that $\gamma$ is known.

Generally, the NTSM control design contains the sliding surface and the reaching law. A discontinuous reaching law with switching function is widely adopted to drive the system trajectory onto the sliding surface, which leads to the chattering. In the following theorem, an exponential-decline switching gain (EDSG) is designed to attenuate the chattering phenomenon.

The EDSG is formulated as

$$
A(t) \alpha_{1}=\left(b_{1}+b_{2} e^{-a t}\right) \alpha_{1},
$$

where $\alpha_{1}>\bar{\varphi}, a>0, b_{1} \geq 1$, and $b_{2}>0$. The EDSG accelerates the convergence rate of sliding surface at the beginning of reaching phase and attenuates the amplitude of chattering without deteriorating the system robustness.

Theorem 2. For system (1), the following controller forces the system dynamic to the sliding surface (6) in finite time:

$$
\begin{aligned}
u= & -\frac{1}{K_{m}}\left(\alpha_{2} s+A(t) \alpha_{1} \operatorname{sign}(s)\right. \\
& \left.+\frac{1}{\beta q}\left|x_{2}\right|^{2-q} \operatorname{sign}(s)\right),
\end{aligned}
$$

where $\alpha_{1}=\bar{\varphi}+\eta, \alpha_{2} \geq 0$, and $\eta$ is a small positive constant.

Proof. Consider the following Lyapunov function for the sliding surface:

$$
V_{1}=\frac{1}{2} s^{2} .
$$

Differentiating (12) with respect to time yields

$$
\begin{aligned}
\dot{V}_{1} & =s \dot{s}=s\left(x_{2}+\beta q\left|x_{2}\right|^{q-1} \dot{x}_{2}\right) \\
& =s\left(x_{2}+\beta q\left|x_{2}\right|^{q-1}(\varphi+\gamma u)\right) .
\end{aligned}
$$


Substituting the controller (11) into (13) yields

$$
\begin{aligned}
\dot{V}_{1} & =s\left(x_{2}+\beta q\left|x_{2}\right|^{q-1}\left(\varphi-\frac{\gamma}{K_{m}}\left(\alpha_{2} s\right.\right.\right. \\
& \left.\left.\left.+A(t) \alpha_{1} \operatorname{sign}(s)+\frac{1}{\beta q}\left|x_{2}\right|^{2-q} \operatorname{sign}(s)\right)\right)\right)=s x_{2} \\
& +\beta q\left|x_{2}\right|^{q-1}\left(\varphi s-\frac{\gamma}{K_{m}} \alpha_{2} s^{2}-\frac{\gamma}{K_{m}} A(t) \alpha_{1}|s|-\frac{\gamma}{K_{m}}\right. \\
& \left.+\frac{1}{\beta q}\left|x_{2}\right|^{2-q}|s|\right)=-\beta q\left|x_{2}\right|^{q-1} \frac{\gamma}{K_{m}} \alpha_{2} s^{2}+s x_{2} \\
& -\frac{\gamma}{K_{m}}|s|\left|x_{2}\right|+\beta q\left|x_{2}\right|^{q-1}\left(\varphi s-\frac{\gamma}{K_{m}} A(t) \alpha_{1}|s|\right) .
\end{aligned}
$$

With $\gamma \geq K_{m}$ and $\left|x_{2}\right|^{q-1}>0$, (14) turns to

$$
\begin{aligned}
\dot{V}_{1} \leq & -\beta q\left|x_{2}\right|^{q-1} \alpha_{2} s^{2}+s x_{2}-|s|\left|x_{2}\right| \\
& +\beta q\left|x_{2}\right|^{q-1}\left(|\varphi||s|-A(t) \alpha_{1}|s|\right) .
\end{aligned}
$$

As $A(t) \alpha_{1} \geq \bar{\varphi}+\eta$, it is obtained as

$$
\begin{aligned}
\dot{V}_{1} & \leq-\beta q\left|x_{2}\right|^{q-1} \alpha_{2} s^{2}-\beta q\left|x_{2}\right|^{q-1} \eta|s| \\
& =-\beta q\left|x_{2}\right|^{q-1}\left(\alpha_{2} s^{2}+\eta|s|\right) \\
& =-\beta q\left|x_{2}\right|^{q-1}\left(2 \alpha_{2} V_{1}+\eta 2^{1 / 2} V_{1}^{1 / 2}\right) .
\end{aligned}
$$

Considering the last expression of (16), if the motion of sliding surface does not stagnate at the points $\left\{x_{1} \neq 0, x_{2}=\right.$ $0\}$ in the reaching phase, it would converge to zero in finite time according to the extended Lyapunov description of finite time stability in Remark 2 of [14].

Here, a demonstration is given to show that the motion of sliding surface does not stagnate at the points $\left\{x_{1} \neq 0, x_{2}=\right.$ $0\}$. obtain

For system (1) with the controller (11), taking $x_{2}=0$, we

$$
\begin{aligned}
\dot{x}_{2} & =\varphi-\frac{\gamma}{K_{m}}\left(\alpha_{2} s+A(t) \alpha_{1} \operatorname{sign}(s)\right. \\
& \left.+\frac{1}{\beta q}\left|x_{2}\right|^{2-q} \operatorname{sign}(s)\right)=-\alpha_{2} \frac{\gamma}{K_{m}} s+\varphi-A(t) \alpha_{1} \\
& \cdot \frac{\gamma}{K_{m}} \operatorname{sign}(s)
\end{aligned}
$$

where $s=x_{1}$. for $\dot{x}_{2}$ :

In the case of $x_{1}>0$, the following inequality is satisfied

$$
\begin{aligned}
\dot{x}_{2} & =-\alpha_{2} \frac{\gamma}{K_{m}} s+\varphi-A(t) \alpha_{1} \frac{\gamma}{K_{m}} \\
& =-\alpha_{2} \frac{\gamma}{K_{m}} s+\varphi-\frac{\gamma}{K_{m}}\left(b_{1}+b_{2} e^{-a t}\right)(\bar{\varphi}+\eta) \\
& \leq \underbrace{-\alpha_{2} s}_{<0}+\underbrace{\varphi-\bar{\varphi}-\eta}_{<0} .
\end{aligned}
$$

In the other case of $x_{1}<0$, it follows that

$$
\begin{aligned}
\dot{x}_{2} & =-\alpha_{2} \frac{\gamma}{K_{m}} s+\varphi+A(t) \alpha_{1} \frac{\gamma}{K_{m}} \\
& =-\alpha_{2} \frac{\gamma}{K_{m}} s+\varphi+\frac{\gamma}{K_{m}}\left(b_{1}+b_{2} e^{-a t}\right)(\bar{\varphi}+\eta) \\
& \geq \underbrace{-\alpha_{2} s}_{>0}+\underbrace{\varphi+\bar{\varphi}+\eta}_{>0} .
\end{aligned}
$$

It is concluded that the points $\left\{x_{1} \neq 0, x_{2}=0\right\}$ are not attractors. Thus, the motion of sliding surface will not stagnate at these points, and it converges to zero in finite time.

This completes the proof.

Remark 3. Note that the term $\left|x_{2}\right|^{2-q} \operatorname{sign}(s)$ of controller (11) is constructed for the stability analysis. It is usually taken as $\left|x_{2}\right|^{2-q} \operatorname{sign}\left(x_{2}\right)$ in the existing NTSM controllers, for example, the controller (8). However, it is not applicable to the stability analysis of the discussed system (1).

Remark 4. In the case that $\gamma$ is known, the proposed controller (11) is also applicable by taking $K_{m}=\gamma$.

\section{Adaptive NTSM Control Scheme Design}

In practical situations, it is usually difficult to obtain the prior knowledge of $\bar{\varphi}$. Therefore, an adaptive controller is designed for system (1) in this section. In the studies of adaptive NTSM control [22, 23], the switching gain was directly adjusted by the adaptive technique. However, it was difficult to prove that the points $\left\{x_{1} \neq 0, x_{2}=0\right\}$ were not attractors as in Theorem 2. So, the adaptive switching gain was modified by the constant control gain in the extreme case that the stagnation problem may happen.

In this section, a double sliding surfaces control scheme is designed to combine the NTSM control with the adaptive technique. The first layer is a common integral sliding mode (ISM) in which the impact of uncertain function $\varphi$ is compensated. The second layer is a NTSM in which the system trajectory converges to the origin. The purpose of using double sliding surfaces is to provide a strict proof for the stagnation problem of NTSM.

In this section, the control law is designed as

$$
u=u_{a}+u_{n}
$$

where $u_{a}$ denotes an adaptive controller which forces the system motion to the first sliding surface. After that, a NTSM controller $u_{n}$ drives the system motion to the second sliding surface.

The first sliding surface ISM is designed as

$$
\begin{aligned}
s_{1} & =x_{2}+z-e^{-\theta t}\left(x_{2}(0)+z(0)\right), \\
\dot{z} & =-u_{n},
\end{aligned}
$$

where $\theta>0$ and $\left\{x_{2}(0), z(0)\right\}$ are the initial values of $\left\{x_{2}, z\right\}$. The exponential-decline term is introduced to eliminate the reaching phase [24]. Then the system dynamic is on the first sliding surface at the initial time. 
Differentiating (21) with respect to time yields

$$
\begin{aligned}
\dot{s}_{1} & =\dot{x}_{2}+\dot{z}+\Lambda=\varphi+\gamma u-u_{n}+\Lambda \\
& =\varphi+\Lambda+(\gamma-1) u_{n}+\gamma u_{a},
\end{aligned}
$$

where $\Lambda=\theta e^{-\theta t}\left(x_{2}(0)+z(0)\right)$. Once the first sliding surface is established, the original system (1) will approach to the system motion of an integral chain system as $\dot{x}_{2}=-u_{n}$.

Here, the NTSM controller $u_{n}$ for system $\dot{x}_{2}=-u_{n}$ is designed as

$$
\begin{aligned}
u_{n}= & -\frac{1}{\beta q}\left|x_{2}\right|^{2-q} \operatorname{sign}\left(x_{2}\right)-\alpha_{2} s_{2} \\
& -\alpha_{3}\left|s_{2}\right|^{p_{1}} \operatorname{sign}\left(s_{2}\right),
\end{aligned}
$$

where $\alpha_{3}$ is a positive constant, $p_{1}$ is a constant satisfying $0<$ $p_{1}<1$, and $s_{2}$ denotes the FNTSM (6).

The continuous controller (23) had been verified in [14], and it also had been proved that the points $\left\{x_{1} \neq 0, x_{2}=\right.$ $0\}$ were not attractors. For an integral chain system without uncertainty (e.g., $\dot{x}_{2}=-u_{n}$ ), the discontinuous control is not necessary. Although the proposed NTSM controller (11) in Theorem 2 can be used to design $u_{n}$, it is not employed as it is discontinuous.

With the following assumption, an adaptive controller is designed in Theorem 6 for system (22) by combining the TSM reaching law with the familiar first-order SMC adaptive control (e.g., [25-27]).

Assumption 5. Assume $\varphi+(\gamma-1) u_{n}$ is unknown and satisfies the following inequality:

$$
\left|\varphi+(\gamma-1) u_{n}\right| \leq c_{0}+c_{1}\left|x_{1}\right|+c_{2}\left|x_{2}\right|
$$

where $c_{0}, c_{1}$, and $c_{2}$ are unknown positive constants.

Theorem 6. For system (22), the sliding surface $s_{1}$ converges to zero asymptotically with the following adaptive controller:

$$
\begin{aligned}
u_{a} & =-\frac{1}{K_{m}}\left(k_{3} s_{1}+k_{4}\left|s_{1}\right|^{p_{2}} \operatorname{sign}\left(s_{1}\right)+|\Lambda| \operatorname{sign}\left(s_{1}\right)\right. \\
& \left.+\left(\widehat{c}_{0}+\widehat{c}_{1}\left|x_{1}\right|+\widehat{c}_{2}\left|x_{2}\right|\right) \operatorname{sign}\left(s_{1}\right)\right), \\
\dot{\hat{c}}_{0} & =k_{0}\left|s_{1}\right|, \\
\dot{\widehat{c}}_{1} & =k_{1}\left|s_{1}\right|\left|x_{1}\right|, \\
\dot{\hat{c}}_{2} & =k_{2}\left|s_{1}\right|\left|x_{2}\right|,
\end{aligned}
$$

where $\left\{k_{0} \sim k_{4}\right\} \geq 0,1 \geq p_{2}>0$, and $\widehat{c}_{i}(0)>0(i=0,1,2)$.

Proof. Define a new Lyapunov function for $s_{1}$ as

$$
V_{2}=\frac{1}{2} s_{1}^{2}+\frac{1}{2 k_{0}} \widetilde{c}_{0}^{2}+\frac{1}{2 k_{1}} \widetilde{c}_{1}^{2}+\frac{1}{2 k_{2}} \widetilde{c}_{2}^{2},
$$

where $\widetilde{c}_{i}=c_{i}-\widehat{c}_{i}$. Its derivative is

$$
\dot{V}_{2}=s_{1} \dot{s}_{1}-\frac{1}{k_{0}} \widetilde{c}_{0} \dot{\widehat{c}}_{0}-\frac{1}{k_{1}} \widetilde{c}_{1} \dot{\hat{c}}_{1}-\frac{1}{k_{2}} \widetilde{c}_{2} \dot{\vec{c}}_{2} .
$$

Substituting controller (25) into (27) yields

$$
\begin{aligned}
\dot{V}_{2} & =s_{1}\left(\varphi+\Lambda+(\gamma-1) u_{n}+\gamma u_{a}\right)-\left(\frac{1}{k_{0}} \widetilde{c}_{0} \dot{\widehat{c}}_{0}\right. \\
& \left.+\frac{1}{k_{1}} \widetilde{c}_{1} \dot{\widehat{c}}_{1}+\frac{1}{k_{2}} \widetilde{c}_{2} \dot{\hat{c}}_{2}\right)=s_{1}\left(\varphi+\Lambda+(\gamma-1) u_{n}\right) \\
& +\gamma s_{1} u_{a}-\left|s_{1}\right|\left(\widetilde{c}_{0}+\widetilde{c}_{1}\left|x_{1}\right|+\widetilde{c}_{2}\left|x_{2}\right|\right) \leq\left|s_{1}\right| \mid \varphi \\
& +(\gamma-1) u_{n}|+| s_{1}|| \Lambda\left|+\gamma s_{1} u_{a}-\right| s_{1} \mid\left(\widetilde{c}_{0}+\widetilde{c}_{1}\left|x_{1}\right|\right. \\
& \left.+\widetilde{c}_{2}\left|x_{2}\right|\right) \leq\left|s_{1}\right|\left(c_{0}+c_{1}\left|x_{1}\right|+c_{2}\left|x_{2}\right|\right)+\left|s_{1}\right||\Lambda| \\
& -\frac{\gamma}{K_{m}}\left(k_{3} s_{1}^{2}+k_{4}\left|s_{1}\right| p_{2}+1+\left|s_{1}\right||\Lambda|\right. \\
& \left.+\left|s_{1}\right|\left(\widehat{c}_{0}+\widehat{c}_{1}\left|x_{1}\right|+\widehat{c}_{2}\left|x_{2}\right|\right)\right)-\left|s_{1}\right|\left(\widetilde{c}_{0}+\widetilde{c}_{1}\left|x_{1}\right|\right. \\
& \left.+\widetilde{c}_{2}\left|x_{2}\right|\right) .
\end{aligned}
$$

With the adaptive laws in $(25)$ and $\widehat{c}_{i}(0)>0$, it ensures that $\widehat{c}_{i}>0$. Since $\gamma \geq K_{m}$, it follows that

$$
\begin{aligned}
\dot{V}_{2} \leq & -\frac{\gamma}{K_{m}}\left(k_{3} s_{1}^{2}+k_{4}\left|s_{1}\right|^{p_{2}+1}\right) \\
& +\left|s_{1}\right|\left(c_{0}+c_{1}\left|x_{1}\right|+c_{2}\left|x_{2}\right|\right) \\
& -\left|s_{1}\right|\left(\widehat{c}_{0}+\widehat{c}_{1}\left|x_{1}\right|+\widehat{c}_{2}\left|x_{2}\right|\right) \\
& -\left|s_{1}\right|\left(\widetilde{c}_{0}+\widetilde{c}_{1}\left|x_{1}\right|+\widetilde{c}_{2}\left|x_{2}\right|\right) \\
= & -\frac{\gamma}{K_{m}}\left(k_{3} s_{1}^{2}+k_{4}\left|s_{1}\right|^{p_{2}+1}\right) .
\end{aligned}
$$

Applying the Barbalat lemma [28], the sliding surface $s_{1}$ converges to zero asymptotically.

This completes the proof.

The boundary layer approach is a common method for chattering reduction. It is well known that the chattering reduction is achieved with a small cost of control precision. More specifically, the sliding surface is driven to a vicinity of zero. In [29], a concept of real sliding surface was introduced which was similar to the boundary layer. It also means that the sliding surface is forced to a small region rather than zero. Based on this concept, a modified adaptive law was proposed to avoid the overestimation of adaptive gain in [29]. The adaptive gain slowly decreased to the necessary value once the real sliding surface was established.

In the case that the boundary layer approach is adopted for the proposed adaptive controller (25), the chattering amplitude can be further attenuated if the adaptive gains $\left\{\widehat{c}_{0}, \widehat{c}_{1}, \widehat{c}_{2}\right\}$ decrease to a low level. Thus, the above two methods are combined to design a modified adaptive controller as

$$
\begin{aligned}
u_{a} & =-\frac{1}{K_{m}}\left(k_{3} s_{1}+k_{4}\left|s_{1}\right|^{p_{2}} \operatorname{sign}\left(s_{1}\right)+|\Lambda| \operatorname{sat}\left(s_{1}\right)\right. \\
& \left.+\left(\widehat{c}_{0}+\widehat{c}_{1}\left|x_{1}\right|+\widehat{c}_{2}\left|x_{2}\right|\right) \operatorname{sat}\left(s_{1}\right)\right)
\end{aligned}
$$




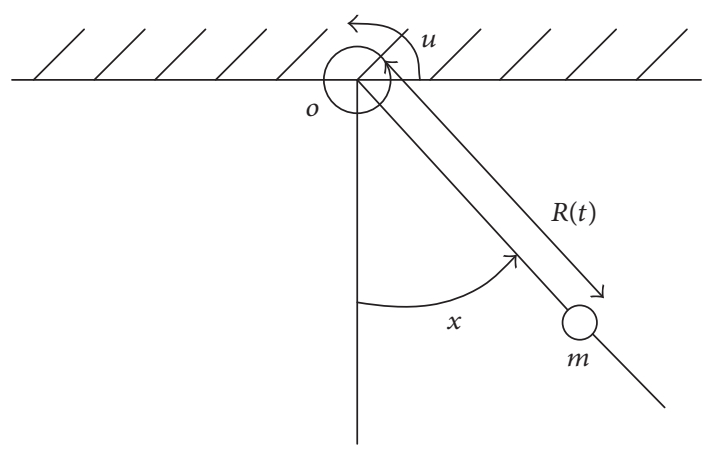

Figure 3: Variable-length pendulum model.

where the sat $(\cdot)$ function and the adaptive laws are taken as

$$
\text { sat } \begin{aligned}
\left(s_{1}\right) & = \begin{cases}\operatorname{sign}\left(s_{1}\right) & \left|s_{1}\right|>O \\
\frac{s_{1}}{O} & \left|s_{1}\right| \leq O,\end{cases} \\
\dot{\vec{c}}_{0} & = \begin{cases}k_{0}\left|s_{1}\right| \operatorname{sign}\left(\left|s_{1}\right|-O\right) & \widehat{c}_{0}>\alpha_{c 0} \\
\bar{k}_{0} & \widehat{c}_{0} \leq \alpha_{c 0},\end{cases} \\
\dot{\vec{c}}_{1} & = \begin{cases}k_{1}\left|s_{1}\right|\left|x_{1}\right| \operatorname{sign}\left(\left|s_{1}\right|-O\right) & \widehat{c}_{1}>\alpha_{c 1} \\
\bar{k}_{1} & \widehat{c}_{1} \leq \alpha_{c 1},\end{cases} \\
\dot{\hat{c}}_{2} & = \begin{cases}k_{2}\left|s_{1}\right|\left|x_{2}\right| \operatorname{sign}\left(\left|s_{1}\right|-O\right) & \widehat{c}_{2}>\alpha_{c 2} \\
\bar{k}_{2} & \widehat{c}_{2} \leq \alpha_{c 2},\end{cases}
\end{aligned}
$$

where a positive constant $O$ denotes the boundary lay. $\left\{\bar{k}_{0} \sim\right.$ $\left.\bar{k}_{2}\right\}$ and $\left\{\alpha_{c 0} \sim \alpha_{c 2}\right\}$ are positive constants.

According to Theorem 6 , the sliding surface $s_{1}$ converges to zero asymptotically. Thus, it converges into $\left|s_{1}\right| \leq O$ in finite time. Then, the adaptive gains begin to decrease, and $\dot{V}_{2}$ becomes sign indefinite. As soon as the sliding surface escapes from the region $\left|s_{1}\right| \leq O$, the adaptive gains increase again so that the sliding surface is driven back into the region $\left|s_{1}\right| \leq O$. Finally, the sliding surface $s_{1}$ remains in a larger region than $\left|s_{1}\right| \leq O$ which is the so-called real sliding surface. The second expressions of the modified adaptive laws ensure the positive values of the adaptive gains, and they are valid in a short time.

Remark 7. For the tracking control issues, it is necessary to modify the adaptive laws of $\widehat{c}_{1}$ and $\widehat{c}_{2}$ to reduce the chattering amplitude. However, it is not necessary when the states $\left\{x_{1}, x_{2}\right\}$ converge to zero.

\section{Simulation}

In this section, the designed robust and adaptive NTSM controllers were tested for the tracking control of a variablelength pendulum [25] (Figure 3) formulated as

$$
\ddot{x}=-2 \frac{\dot{R}(t)}{R(t)} \dot{x}-\frac{g}{R(t)} \sin (x)+\frac{1}{m R^{2}(t)} u .
$$

The angular coordinate $x$ is impelled to track a reference signal $x_{c}$ by the torque $u$. A known mass $m$ moves alone the rod without friction, and the distance $R(t)$ between the center $o$ and the mass $m$ is unmeasured. It is assumed that the states $\left[\begin{array}{ll}x & \dot{x}\end{array}\right]^{T}$ can be measured for the controller design. The parameters of (32) and the reference signal are taken as

$$
\begin{aligned}
R(t) & =1+0.25 \sin (4 t)+0.5 \cos (t) \\
m & =1 \mathrm{~kg}, \\
g & =9.81 \mathrm{~m} / \mathrm{s}^{2}, \\
x(0) & =1.2, \\
x_{c} & =0.5 \sin (0.5 t) .
\end{aligned}
$$

Defining the tracking error as $e_{1}=x-x_{c}$, the tracking control is converted to the stabilization of the following error system:

$$
\begin{aligned}
& \dot{e}_{1}=e_{2}, \\
& \dot{e}_{2}=-2 \frac{\dot{R}(t)}{R(t)} \dot{x}-\frac{g}{R(t)} \sin (x)-\ddot{x}_{c}+\frac{1}{m R^{2}(t)} u .
\end{aligned}
$$

4.1. Robust Control. In this subsection, three robust controllers (A, B, and C) were evaluated. According to Theorem 2, controllers A and B (without EDSG) were designed using the FNTSM of [11] and the proposed FNTSM (6), respectively. The controller C was the proposed FNTSM-EDSG controller in Theorem 2.

Controller A (based on FNTSM of [11]):

$$
\begin{aligned}
u= & -16\left(0.2 s_{F 1}+\alpha_{1} \operatorname{sign}\left(s_{F 1}\right)\right. \\
& +\frac{7}{90} e_{2}^{5 / 7} \operatorname{sign}\left(s_{F 1}\right) \operatorname{sign}\left(e_{2}\right) \\
& \left.+\frac{49}{450} e_{1}^{2 / 5} e_{2}^{5 / 7} \operatorname{sign}\left(s_{F 1}\right) \operatorname{sign}\left(e_{1}^{2 / 5} e_{2}\right)\right), \\
& s_{F 1}=e_{1}+e_{1}^{7 / 5}+e_{2}^{9 / 7}, \alpha_{1}=0.4+0.4\left|x_{1}\right|+0.4\left|x_{2}\right| .
\end{aligned}
$$

Controller B (based on FNTSM (6)):

$$
\begin{aligned}
& u=-16\left(0.2 s_{F 2}+\alpha_{1} \operatorname{sign}\left(s_{F 2}\right)+\frac{1}{q}\left|e_{2}\right|^{2-q} \operatorname{sign}\left(s_{F 2}\right)\right), \\
& s_{F 2}=e_{1}+\left|e_{2}\right|^{q} \operatorname{sign}\left(e_{2}\right), q=\frac{9}{7}+\left(1-\frac{9}{7}\right) \operatorname{sign}\left(\left|x_{1}\right|-1\right) .
\end{aligned}
$$

Controller C (based on FNTSM (6) + EDSG):

$$
\begin{aligned}
& u=-16\left(0.2 s_{F 2}+A(t) \alpha_{1} \operatorname{sign}\left(s_{F 2}\right)\right. \\
& \left.+\frac{1}{q}\left|e_{2}\right|^{2-q} \operatorname{sign}\left(s_{F 2}\right)\right), \\
& \quad A(t)=1+e^{-2 t}, \alpha_{1}=0.2+0.2\left|x_{1}\right|+0.2\left|x_{2}\right| .
\end{aligned}
$$

The simulation results are given in Figure 4. As shown in Figures 4(a) and 4(b), the angular coordinate converged 

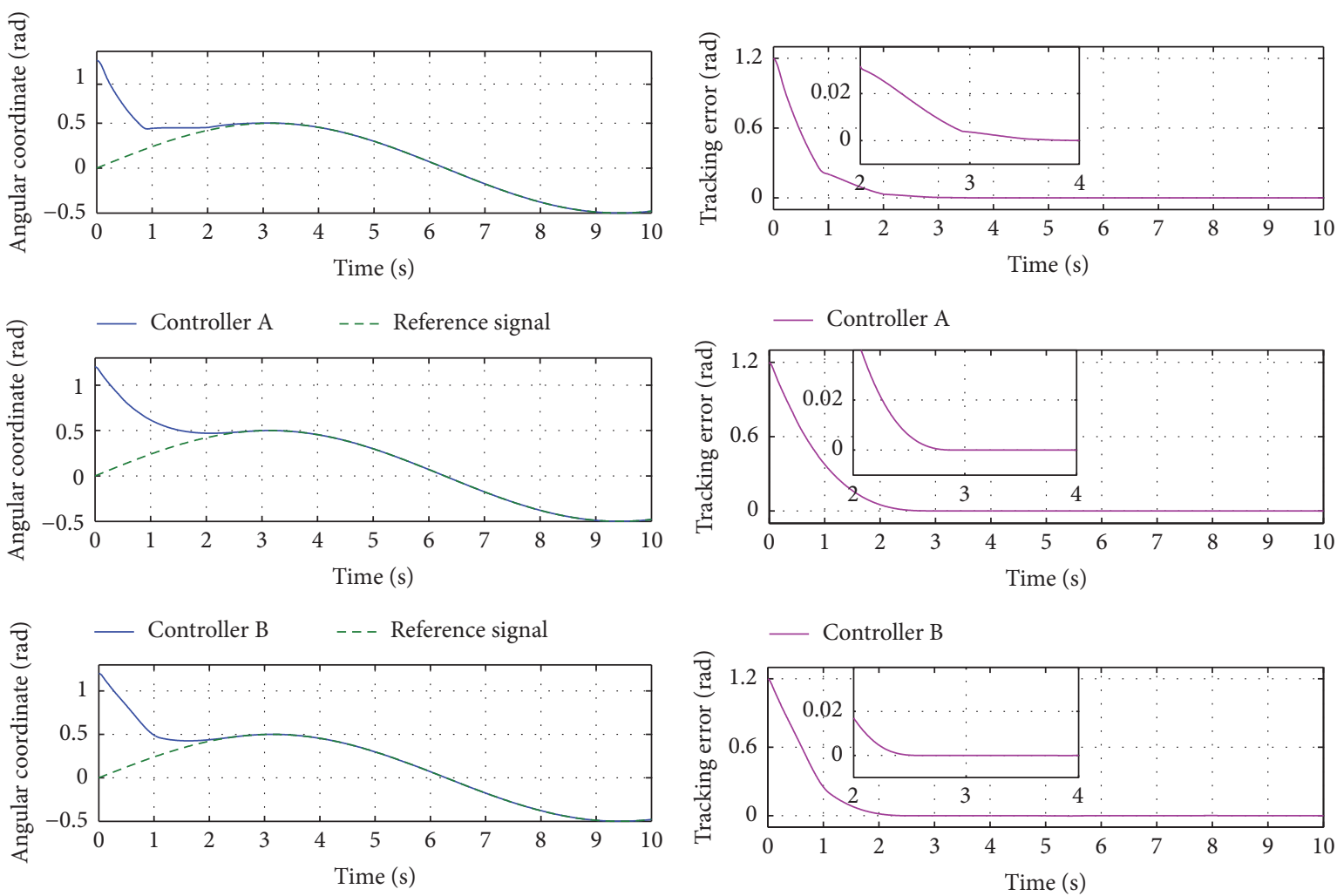

— Controller C

- - - Reference signal

(a) Angular coordinate

- Controller C

(b) Tracking error
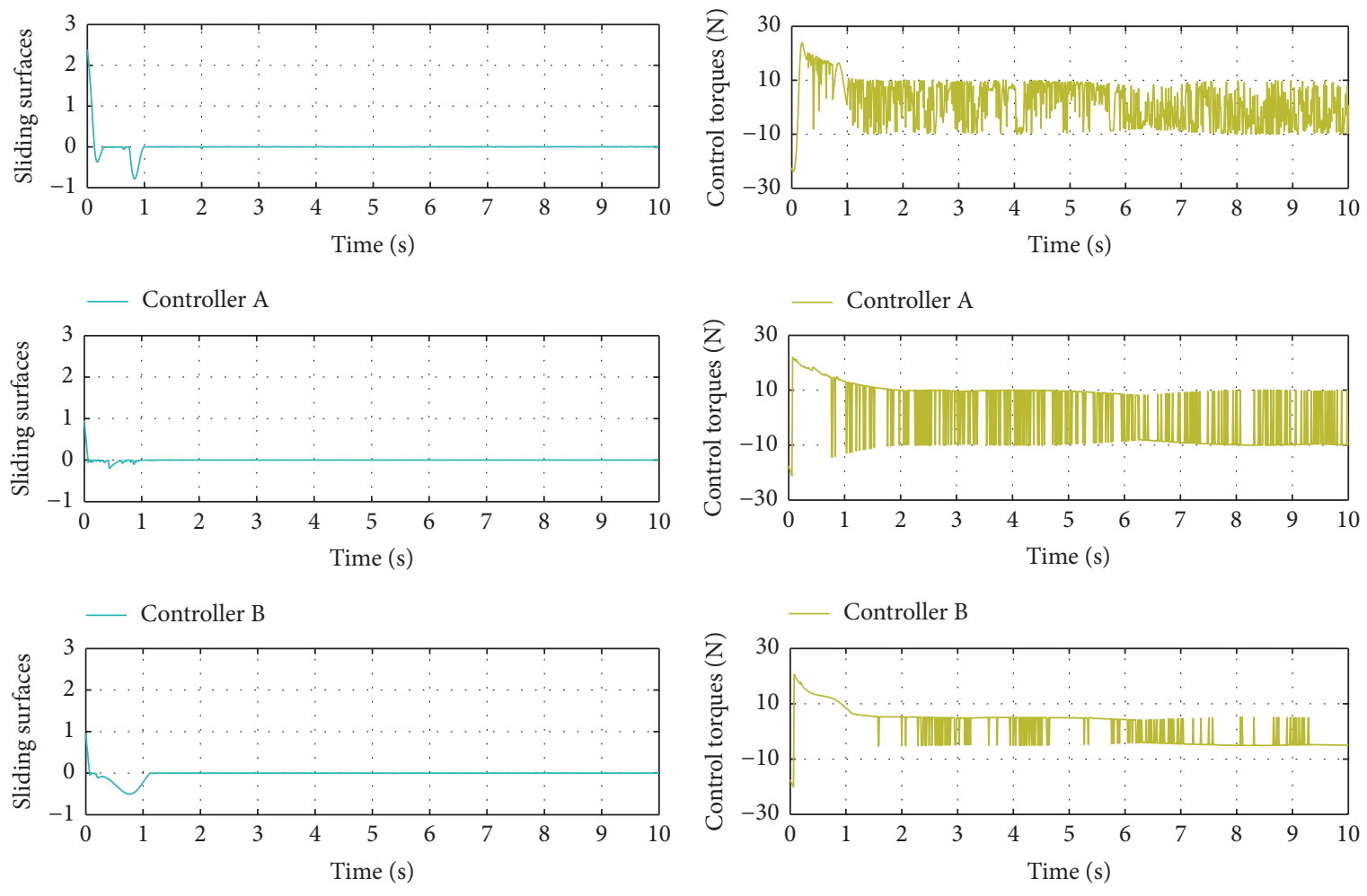

- Controller C

(c) Sliding surfaces

(d) Control torque

FIGURE 4: Simulation result of the robust control. 
to the reference signal within $t=4 \mathrm{~s}$. The sliding surfaces converged to zero within $t=1.5 \mathrm{~s}$ (Figure 4(c)). It is clear that controller B cost less convergence time than the controller A, while the control inputs of these controllers had slight difference (Figures 4(b) and 4(d)). This fact implies that the controller designed by the proposed FNTSM was more efficient. As expected, the chattering amplitude of controller C $( \pm 5)$ was half of controller B $( \pm 10)$ (Figure $4(d)$ ). However, the performance of controller $\mathrm{C}$ had small difference with that of controller B. The result confirms that the EDSG is effective for chattering suppression without deteriorating the system robustness.

4.2. Adaptive Control. In this subsection, the proposed adaptive NTSM control scheme ((23) and (25)) was verified for the tracking control. As a comparison, the adaptive method in [25] was adopted to design the adaptive controller $u_{a}$. The controllers were designed as follows.

Controller D (Theorem 6):

$$
\begin{aligned}
u_{n} & =-\frac{1}{q}\left|e_{2}\right|^{2-q} \operatorname{sign}\left(e_{2}\right)-2.5 s_{2}-\left|s_{2}\right|^{7 / 9} \operatorname{sign}\left(s_{2}\right) \\
u_{a} & =-16\left(0.5 s_{1}+\left|s_{1}\right|^{7 / 9} \operatorname{sign}\left(s_{1}\right)\right. \\
& +\left|5 e^{-5 t}\left(e_{2}(0)+z(0)\right)\right| \operatorname{sign}\left(s_{1}\right) \\
& \left.+\left(\widehat{c}_{0}+\widehat{c}_{1}\left|x_{1}\right|+\widehat{c}_{2}\left|x_{2}\right|\right) \operatorname{sign}\left(s_{1}\right)\right) \\
s_{1} & =e_{2}+z-e^{-5 t}\left(e_{2}(0)+z(0)\right) \\
\dot{z} & =-u_{n}, \\
z & (0)=0 \\
\dot{\widehat{c}}_{0}= & \left|s_{1}\right| \\
\dot{\widehat{c}}_{1} & =\left|s_{1}\right|\left|x_{1}\right| \\
\dot{\hat{c}}_{2} & =\left|s_{1}\right|\left|x_{2}\right| \\
\widehat{c}_{0} & (0)=\widehat{c}_{1}(0)=\widehat{c}_{2}(0)=0.01 .
\end{aligned}
$$

Controller E (method in [25]):

$$
\begin{aligned}
& u_{a}=-5 e^{-5 t}\left(e_{2}(0)+z(0)\right)-\widehat{c}_{0} \operatorname{sign}\left(s_{1}\right), \\
& \dot{\widehat{c}}_{0}=5\left|s_{1}\right| .
\end{aligned}
$$

Controllers D and E shared the same NTSM controller $u_{n}$, and the sliding surface $s_{2}$ was taken as $s_{F 2}$ of controller $\mathrm{B}$.

The simulation results are given in Figures 5 and 6. It is clear that both controllers D and $\mathrm{E}$ achieved fast tracking of the reference signal. Controller D had shorter convergence time and better transient performance than controller E, even though the maximum control torque and the chattering amplitude of controller D were smaller. Meanwhile, the sliding surfaces in Figure 6 converged to zero within $t=$ 3 s. In Figure 6(a), the motions of ISM started from zero, and there was a short time that ISM did not remain zero due to the affection of uncertainty. It is observed that the
FNTSM converged to zero after the ISM, and the stagnation of FNTSM never happened. It verifies that the designed adaptive NTSM control scheme is effective. Since the two controllers shared the same NTSM controller $u_{n}$, the simulation results confirm that the proposed adaptive method had better transient performance and was more efficient than the method in [25].

Here, the modified adaptive controller ((30) and (31)) was checked for chattering reduction. In the following controller F, the boundary layer approach and the modified adaptive laws were both employed. As a comparison, controller G only employed the boundary layer approach. In order to avoid the unbounded growing of adaptive gains inside the boundary layer, the switching adaptive laws were adopted for controller $G$. In addition, controllers $F$ and $G$ used the same boundary layer and NTSM controller $u_{n}$ of controller D. Since it is assumed that the states can be measured for the controller design, the measurement noise may deteriorate the performance of controller in real implementations. To illustrate the robustness of the controller, random noise was taken for the states $\left[\begin{array}{ll}x & \dot{x}\end{array}\right]^{T}$ (mean value 0 and standard deviation 0.01).

Controller F:

$$
\begin{aligned}
u_{a} & =-16\left(0.5 s_{1}+\left|s_{1}\right|^{7 / 9} \operatorname{sign}\left(s_{1}\right)\right. \\
& +\left|5 e^{-5 t}\left(e_{2}(0)+z(0)\right)\right| \operatorname{sat}\left(s_{1}\right) \\
& \left.+\left(\widehat{c}_{0}+\widehat{c}_{1}\left|x_{1}\right|+\widehat{c}_{2}\left|x_{2}\right|\right) \operatorname{sat}\left(s_{1}\right)\right), \\
\operatorname{sat} & \left(s_{1}\right)= \begin{cases}\operatorname{sign}\left(s_{1}\right) & \left|s_{1}\right|>0.04 \\
\frac{s_{1}}{0.04} & \left|s_{1}\right| \leq 0.04,\end{cases} \\
\dot{\vec{c}}_{0} & = \begin{cases}2\left|s_{1}\right| \operatorname{sign}\left(\left|s_{1}\right|-0.04\right) & \widehat{c}_{0}>0.01 \\
1 & \widehat{c}_{0} \leq 0.01,\end{cases} \\
\dot{\vec{c}}_{1} & = \begin{cases}2\left|s_{1}\right|\left|x_{1}\right| \operatorname{sign}\left(\left|s_{1}\right|-0.05\right) & \widehat{c}_{1}>0.01 \\
1 & \widehat{c}_{1} \leq 0.01,\end{cases} \\
\dot{\widehat{c}}_{2} & = \begin{cases}2\left|s_{1}\right|\left|x_{2}\right| \operatorname{sign}\left(\left|s_{1}\right|-0.04\right) & \widehat{c}_{2}>0.01 \\
1 & \widehat{c}_{2} \leq 0.01 .\end{cases}
\end{aligned}
$$

Controller G:

$$
\begin{aligned}
u_{a} & =-16\left(0.5 s_{1}+\left|s_{1}\right|^{7 / 9} \operatorname{sign}\left(s_{1}\right)\right. \\
& +\left|5 e^{-5 t}\left(e_{2}(0)+z(0)\right)\right| \operatorname{sat}\left(s_{1}\right) \\
& \left.+\left(\widehat{c}_{0}+\widehat{c}_{1}\left|x_{1}\right|+\widehat{c}_{2}\left|x_{2}\right|\right) \operatorname{sat}\left(s_{1}\right)\right), \\
\dot{\vec{c}}_{0} & = \begin{cases}2\left|s_{1}\right| & s_{1}>0.04 \\
0 & s_{1} \leq 0.04,\end{cases}
\end{aligned}
$$




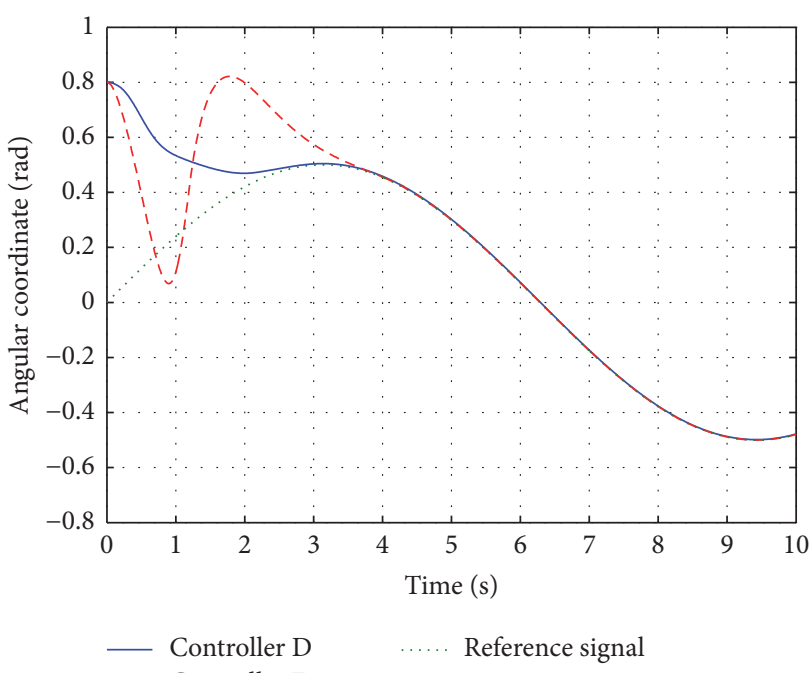

- - - Controller E

(a) Angular coordinate

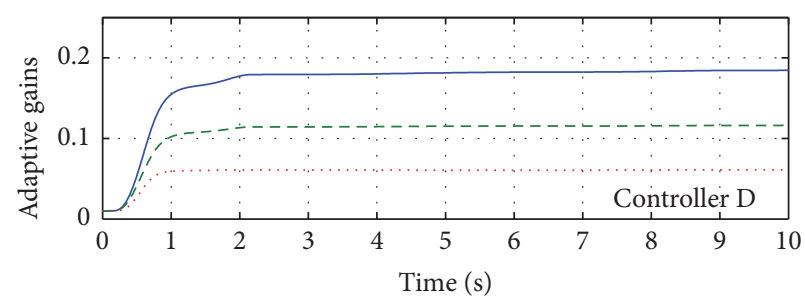

- Adaptive gain 1

- - - Adaptive gain 2

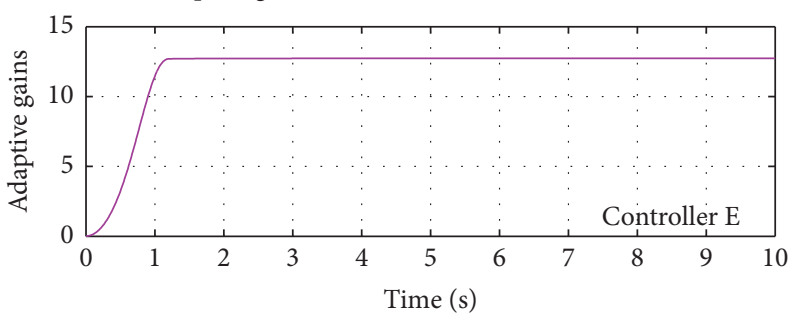

— Adaptive gain 1

(c) Adaptive gains

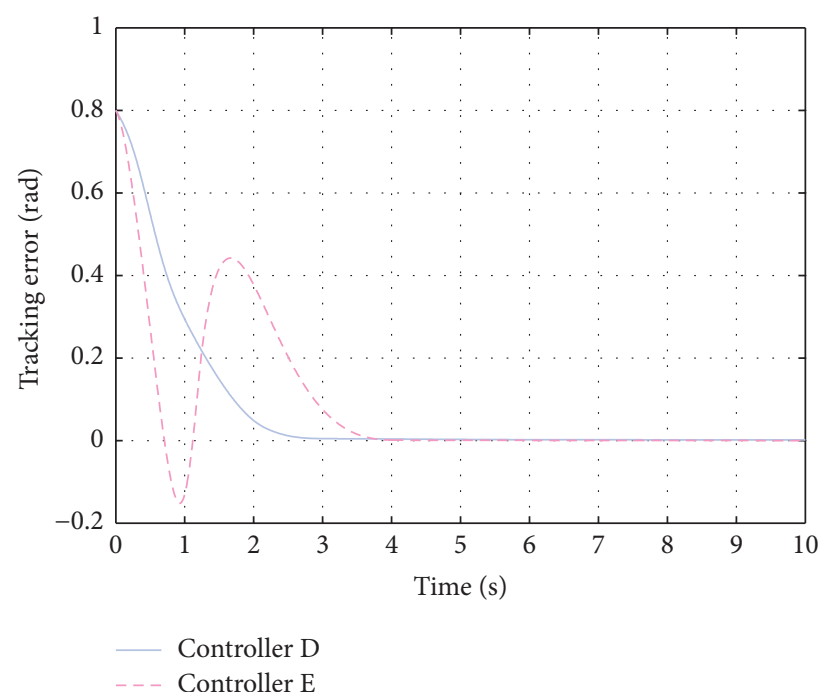

(b) Tracking error

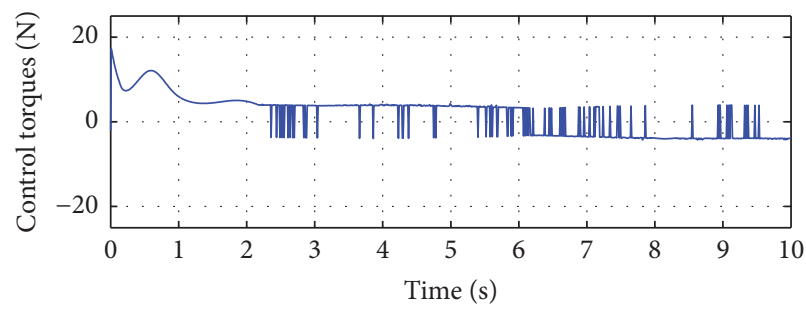

— Controller D

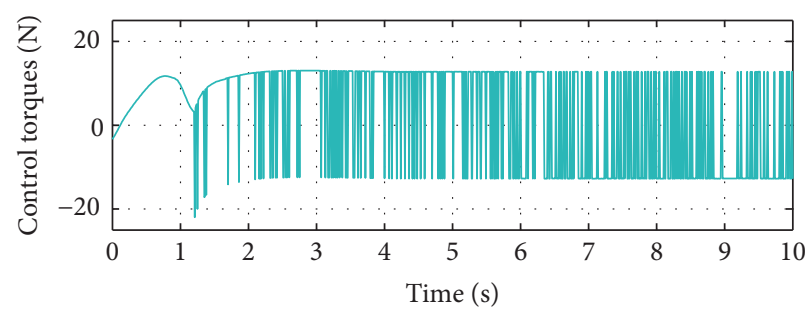

_ Controller E

(d) Control torque

FIGURE 5: Simulation result of the adaptive control.

$$
\begin{aligned}
& \dot{\vec{c}}_{1}= \begin{cases}2\left|s_{1}\right|\left|x_{1}\right| & s_{1}>0.04 \\
0 & s_{1} \leq 0.04\end{cases} \\
& \dot{\hat{c}}_{2}= \begin{cases}2\left|s_{1}\right|\left|x_{2}\right| & s_{1}>0.04 \\
0 & s_{1} \leq 0.04\end{cases}
\end{aligned}
$$

The simulation result of controller $\mathrm{F}$ is given in Figure 7. It is shown that the angular coordinate tracked the reference signal with a high precision (Figure $7(\mathrm{a})$ ). Due to the adoption of the boundary layer and the modified adaptive laws, the sliding surface $s_{1}$ converged to a small domain as $\left|s_{1}\right| \leq 0.1$
(Figure 7(b)). Moreover, the adaptive gains decreased to a low level with approximate reduction ratios as $51.08 \%, 84.79 \%$, and $16.15 \%$, respectively (Figure $7(\mathrm{c})$ ). As a result, there was a marked reduction in the chattering of controller F compared to that of controller G in Figure 7(d). Thus, the effectiveness of the modified adaptive controller is verified.

\section{Conclusions}

This paper studies the NTSM control for a class of secondorder systems in which the coefficient of control input is unknown. In this paper, a new FNTSM was designed to integrate the advantages of LSM and NTSM. Based on this FNTSM, new forms of robust controller and adaptive 


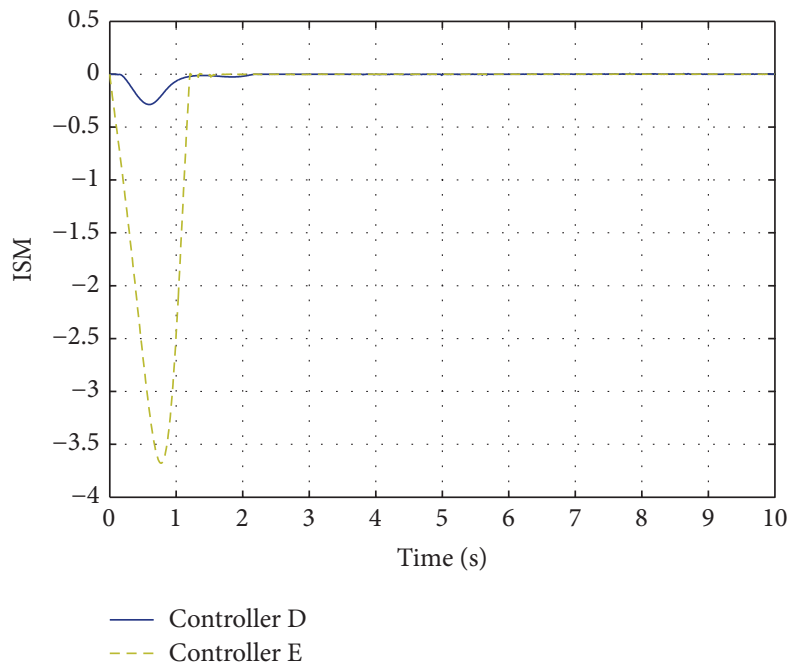

(a) ISM

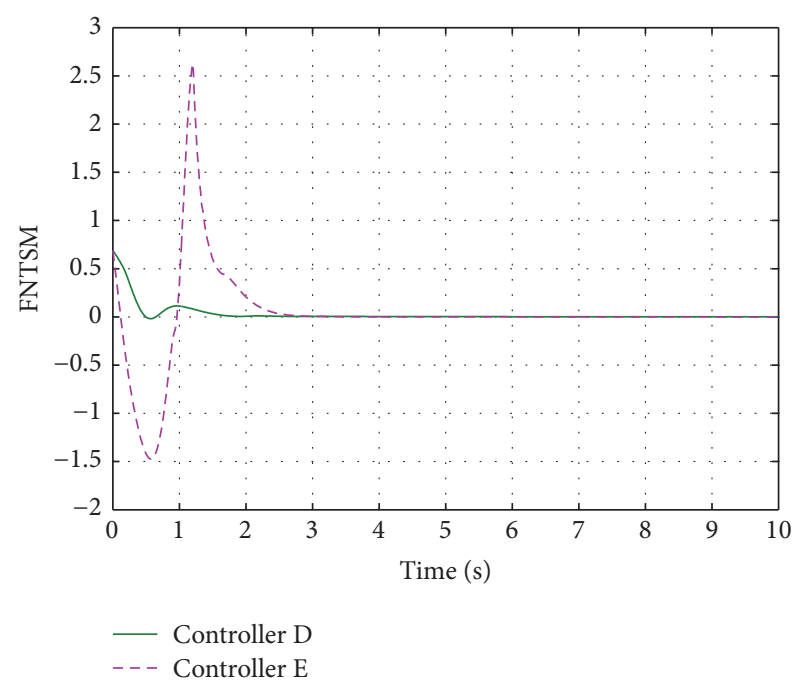

(b) FNTSM

FIGURE 6: The response of sliding surfaces.

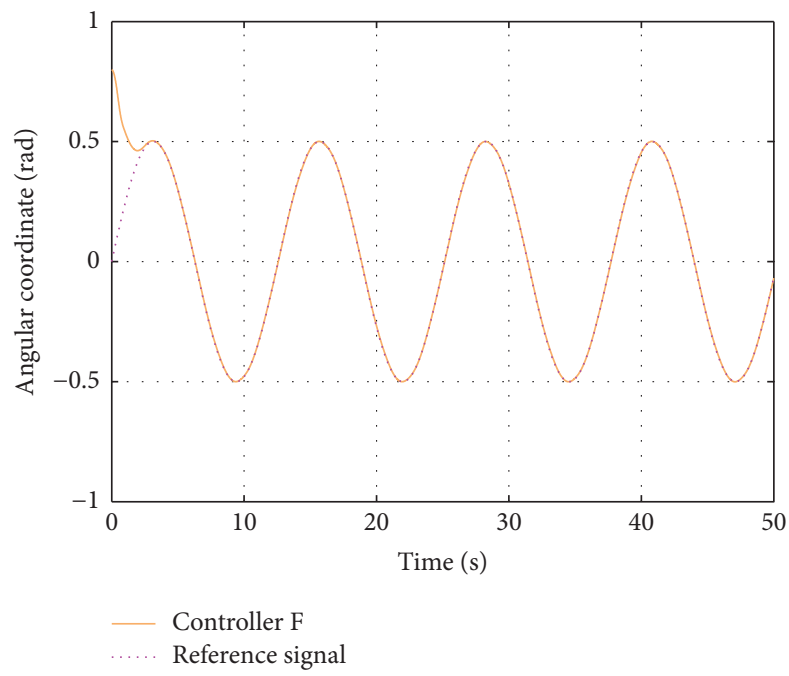

(a) Angular coordinate

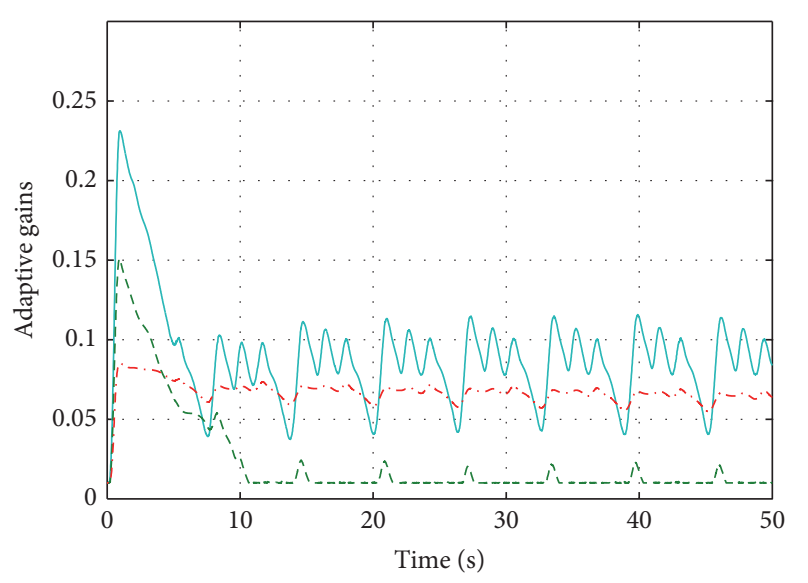

— Adaptive gain $1 \quad \ldots$ Adaptive gain 3

- - - Adaptive gain 2

(c) Adaptive gains

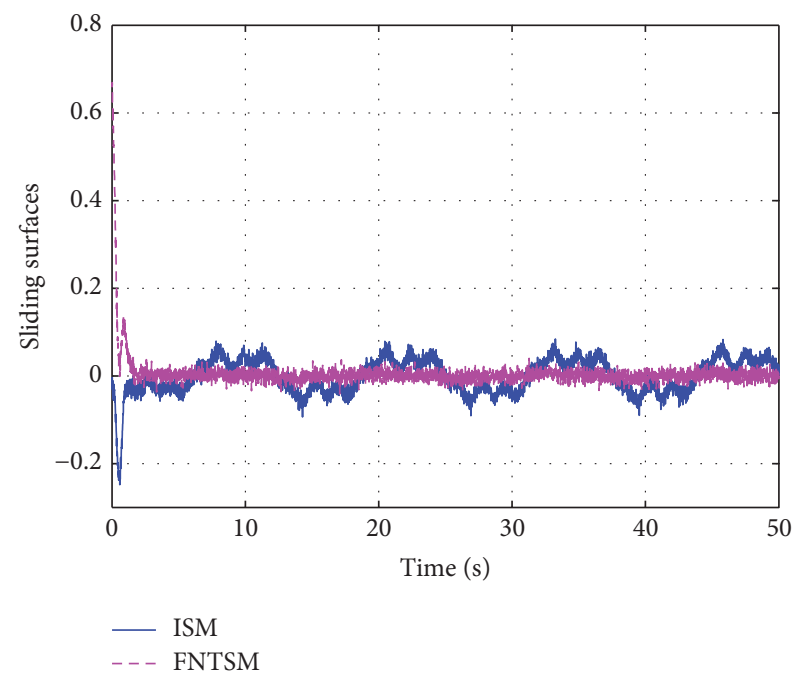

(b) Sliding surfaces
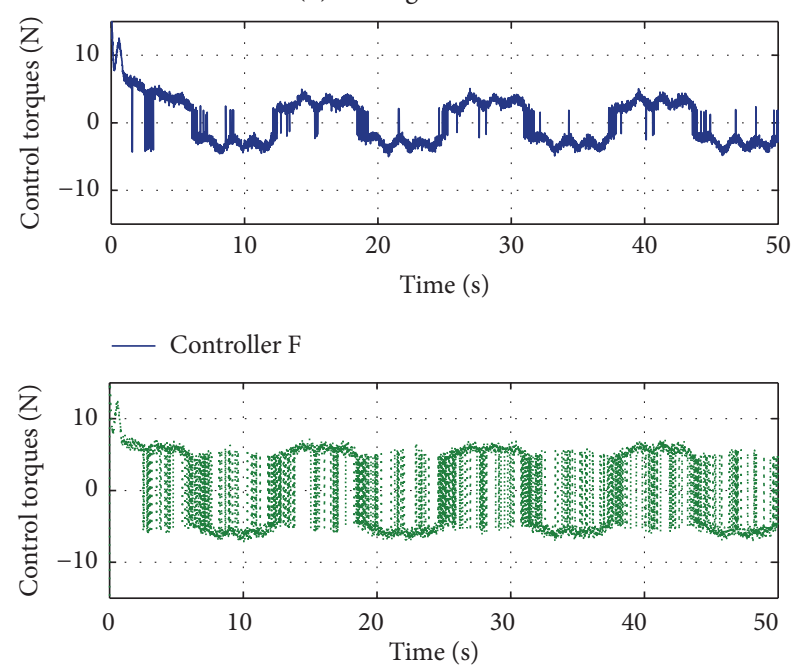

Controller G

(d) Control torques

FIGURE 7: Simulation result of the modified adaptive control. 
controller were proposed for the discussed uncertain system. The simulation verified the effectiveness of the proposed controllers. The proposed FNTSM shows faster convergence rate than the NTSM, and chattering reduction was achieved by the EDSG. The adaptive controller presented better transient performance and more efficiency than the adaptive method in [25]. Moreover, the modified adaptive controller achieved effective chattering reduction.

\section{Competing Interests}

The authors declare that there is no conflict of interests regarding the publication of this article.

\section{Acknowledgments}

This research is supported by National Natural Science Foundation of China (no. 61375100, no. 61472037, and no. 61433003).

\section{References}

[1] J. M. Zhang, C. Y. Sun, R. M. Zhang, and C. Qian, "Adaptive sliding mode control for re-entry attitude of near space hypersonic vehicle based on backstepping design," IEEE/CAA Journal of Automatica Sinica, vol. 2, no. 1, pp. 94-101, 2015.

[2] J. Liu, Y. Zhao, B. Geng, and B. Xiao, "Adaptive second order sliding mode control of a fuel cell hybrid system for electric vehicle applications," Mathematical Problems in Engineering, vol. 2015, Article ID 370424, 14 pages, 2015.

[3] M. Zak, "Terminal attractors in neural networks," Neural Networks, vol. 2, no. 4, pp. 259-274, 1989.

[4] S. T. Venkataraman and S. Gulati, "Terminal sliding modes: a new approach to nonlinear control synthesis," in Proceedings of the 5th International Conference on Advanced Robotics, vol. 1, pp. 443-448, Pisa, Italy, June 1991.

[5] D. Y. Zhao, S. Y. Li, and F. Gao, "A new terminal sliding mode control for robotic manipulators," International Journal of Control, vol. 82, no. 10, pp. 1804-1813, 2009.

[6] Z. K. Song, H. X. Li, and K. B. Sun, "Finite-time control for nonlinear spacecraft attitude based on terminal sliding mode technique," ISA Transactions, vol. 53, no. 1, pp. 117-124, 2014.

[7] H. Komurcugil, "Non-singular terminal sliding-mode control of DC-DC buck converters," Control Engineering Practice, vol. 21, no. 3, pp. 321-332, 2013.

[8] X. Yu, M. Zhihong, and Y. Wu, "Terminal sliding modes with fast transient performance," in Proceedings of the 1997 36th IEEE Conference on Decision and Control. Part 1 (of 5), vol. 2, pp. 962963, San Diego, Calif, USA, December 1997.

[9] S. Mobayen, "Fast terminal sliding mode controller design for nonlinear second-order systems with time-varying uncertainties," Complexity, vol. 21, no. 2, pp. 239-244, 2015.

[10] Z. He, C. Liu, Y. Zhan, H. Li, X. Huang, and Z. Zhang, "Nonsingular fast terminal sliding mode control with extended state observer and tracking differentiator for uncertain nonlinear systems," Mathematical Problems in Engineering, vol. 2014, Article ID 639707, 16 pages, 2014.

[11] L. Liu, Q. M. Zhu, L. Cheng et al., "Applied methods and techniques for mechatronic systems: modelling, identification and control," Lecture Notes in Control and Information Sciences, vol. 452, pp. 79-97, 2014.

[12] X. H. Yu and Z. H. Man, "On finite time mechanism: terminal sliding modes", in Proceedings of the IEEE International Workshop on Variable Structure Systems, pp. 164-167, Tokyo, Japan, 1996.

[13] L. Y. Wang, T. Y. Chai, and L. F. Zhai, "Neural-network-based terminal sliding-mode control of robotic manipulators including actuator dynamics," IEEE Transactions on Industrial Electronics, vol. 56, no. 9, pp. 3296-3304, 2009.

[14] S. Yu, X. Yu, B. Shirinzadeh, and Z. Man, "Continuous finitetime control for robotic manipulators with terminal sliding mode," Automatica, vol. 41, no. 11, pp. 1957-1964, 2005.

[15] A. Al-Ghanimi, J. Zheng, and Z. Man, "Robust and fast nonsingular terminal sliding mode control for piezoelectric actuators," IET Control Theory \& Applications, vol. 9, no. 18, pp. 26782687, 2015.

[16] S. Mobayen, "Finite-time tracking control of chained-form nonholonomic systems with external disturbances based on recursive terminal sliding mode method," Nonlinear Dynamics, vol. 80, no. 1-2, pp. 669-683, 2015.

[17] S. Mobayen, "Fast terminal sliding mode tracking of nonholonomic systems with exponential decay rate," IET Control Theory \& Applications, vol. 9, no. 8, pp. 1294-1301, 2015.

[18] Y. Wu and J. Wang, "Continuous recursive sliding mode control for hypersonic flight vehicle with extended disturbance observer," Mathematical Problems in Engineering, vol. 2015, Article ID 506906, 26 pages, 2015.

[19] S. Mobayen, "An adaptive fast terminal sliding mode control combined with global sliding mode scheme for tracking control of uncertain nonlinear third-order systems," Nonlinear Dynamics, vol. 82, no. 1-2, pp. 599-610, 2015.

[20] N. Boonsatit and C. Pukdeboon, "Adaptive fast terminal sliding mode control of magnetic levitation system," Journal of Control, Automation and Electrical Systems, vol. 27, no. 4, pp. 359-367, 2016.

[21] L. Y. Fang, T. S. Li, Z. F. Li, and R. Li, "Adaptive terminal sliding mode control for anti-synchronization of uncertain chaotic systems," Nonlinear Dynamics, vol. 74, no. 4, pp. 991-1002, 2013.

[22] C.-C. Yang, "Synchronization of second-order chaotic systems via adaptive terminal sliding mode control with input nonlinearity," Journal of the Franklin Institute. Engineering and Applied Mathematics, vol. 349, no. 6, pp. 2019-2032, 2012.

[23] C.-C. Yang and C.-J. Ou, "Adaptive terminal sliding mode control subject to input nonlinearity for synchronization of chaotic gyros," Communications in Nonlinear Science and Numerical Simulation, vol. 18, no. 3, pp. 682-691, 2013.

[24] J.-J. Kim, J.-J. Lee, K.-B. Park, and M.-J. Youn, "Design of new time-varying sliding surface for robot manipulator using variable structure controller," Electronics Letters, vol. 29, no. 2, pp. 195-196, 1993.

[25] P. Li and Z.-Q. Zheng, "Robust adaptive second-order slidingmode control with fast transient performance," IET Control Theory \& Applications, vol. 6, no. 2, pp. 305-312, 2012.

[26] M. Zhihong, M. O'Day, and X. Yu, "A robust adaptive terminal sliding mode control for rigid robotic manipulators," Journal of Intelligent and Robotic Systems: Theory and Applications, vol. 24, no. 1, pp. 23-41, 1999.

[27] Z. Zhu, Y. Xia, and M. Fu, "Adaptive sliding mode control for attitude stabilization with actuator saturation," IEEE Transactions on Industrial Electronics, vol. 58, no. 10, pp. 4898-4907, 2011. 
[28] H. K. Khalil, Nonlinear Systems, Prentice Hall, Englewood Cliffs, NJ, USA, 3rd edition, 2002.

[29] Y. Shtessel, M. Taleb, and F. Plestan, "A novel adaptive-gain supertwisting sliding mode controller: methodology and application," Automatica, vol. 48, no. 5, pp. 759-769, 2012. 


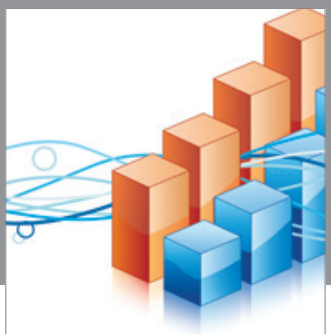

Advances in

Operations Research

vatem alat4

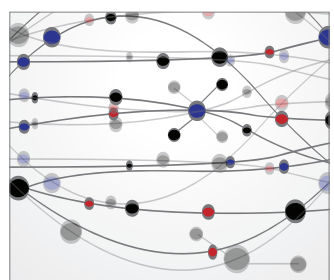

\section{The Scientific} World Journal
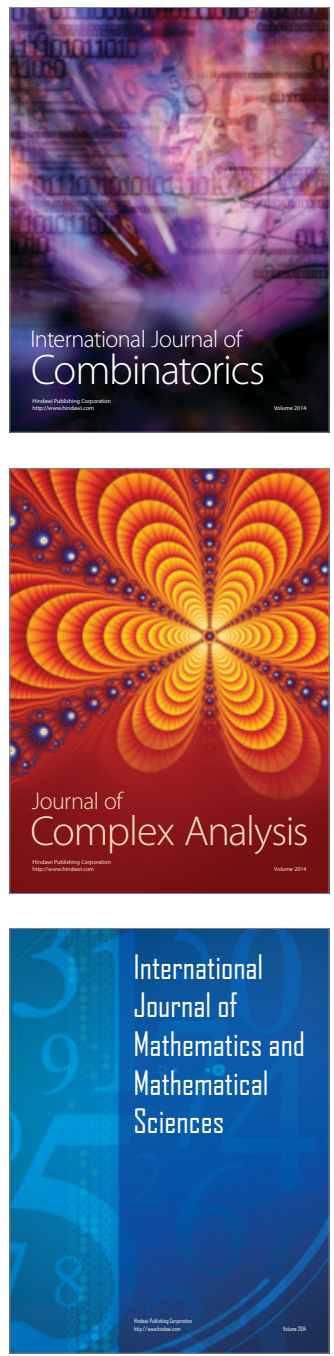
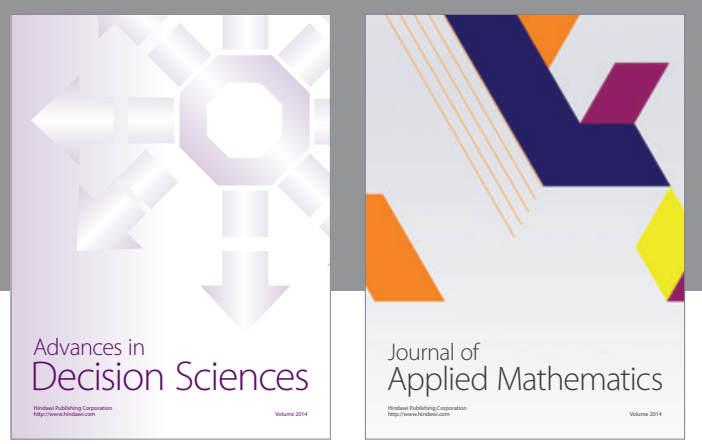

Algebra

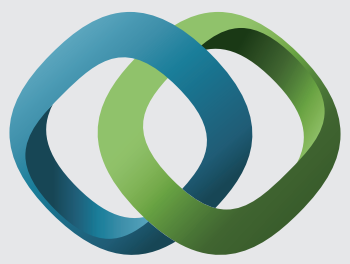

\section{Hindawi}

Submit your manuscripts at

http://www.hindawi.com
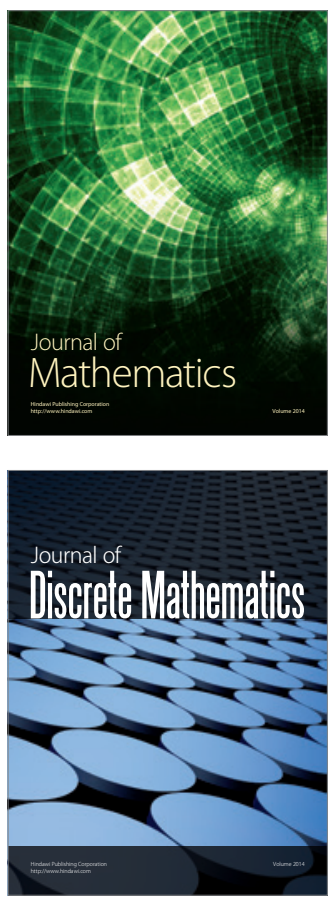

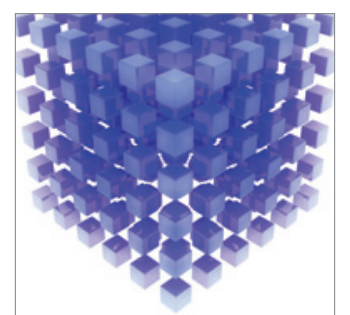

Mathematical Problems in Engineering
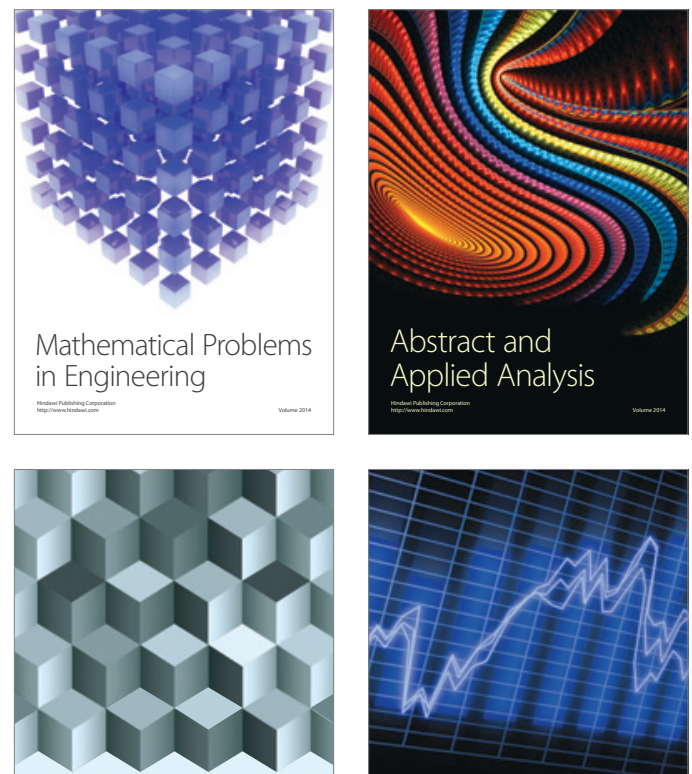

Journal of

Function Spaces

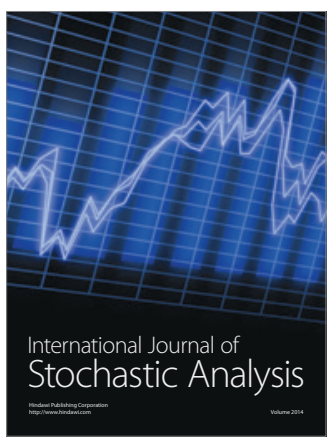

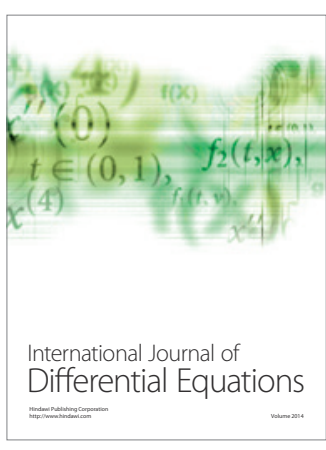
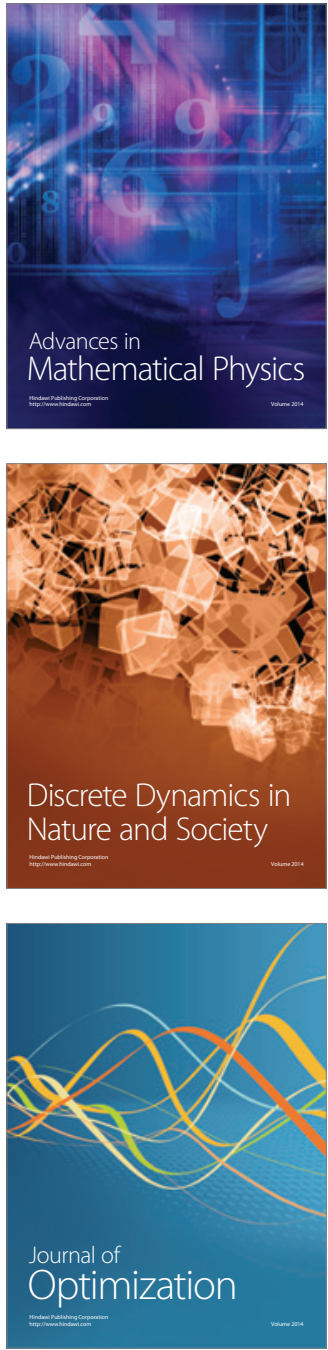